[Frontiers in Bioscience 14, 887-899, January 1, 2009]

\title{
Application of RGD-containing peptides as imaging probes for alphavbeta3 expression
}

Ingrid Dijkgraaf ${ }^{1}$, Ambros J. Beer ${ }^{1}$ and Hans-Jurgen Wester ${ }^{1}$

${ }^{I}$ Technische Universitat Munchen, Department of Nuclear Medicine, Klinikum rechts der Isar, 81675 Munchen, Germany

\section{TABLE OF CONTENTS}

1. Abstract

2. Introduction

3. $R G D$-containing peptides as imaging probes for alphavbeta 3 expression 3.1 Nuclear imaging

3.1.1. Monomeric radiolabeled RGD peptides 3.1.2. RGD- multimers

3.2 Magnetic resonance imaging

3.3 Ultrasound

3.4 Optical imaging of alphavbeta 3 expression

4. Translation into the clinic

5. Conclusions

6. References

\section{ABSTRACT}

Integrin alphavbeta3 plays a pivotale role in tumor angiogenesis and is a receptor for the extracellular matrix proteins with the exposed arginine-glysine-aspartic acid (RGD) tripeptide sequence (e.g. vitronectin, fibronectin). Alphavbeta 3 is overexpressed on activated endothelial cells during tumor-induced angiogenesis, whereas it is absent on quiescent endothelial cells and normal tissues. Furthermore, alphavbeta 3 is expressed on various tumor cell lines. Due to this restricted expression of alphavbeta 3 in tumors, alphavbeta3 is considered a suitable receptor for tumor targeting. In the past decade, several RGD-containing peptide antagonists have been evaluated for monitoring alphavbeta3 expression using SPECT, PET, MRI, OI and US. Molecular imaging tracers for this integrin receptor could be used to noninvasively visualize alphavbeta3 expression in tumors. Noninvasive determination of alphavbeta3 expression potentially can be used to monitor treatment response to antiangiogenic drugs or even to select patients likely to respond to treatment with antiangiogenic drugs. In this review a brief overview on the currently used RGD-containing peptides as imaging probes for noninvasive visualization of alphavbeta3 expression using PET, SPECT, MRI, OI and US is given.

\section{INTRODUCTION}

Integrins are a family of transmembrane glycoproteins that function in cellular adhesion, migration and signal transduction. The term "integrin" was derived from the ability of these proteins to link the extracellular matrix (ECM) proteins with the intracellular cytoskeleton (1). Each member of this family consists of two noncovalently bound transmembrane polypeptide subunits, alpha and beta. Integrins have a large extracellular domain which binds extracellular ligands, a transmembrane domain, and a relatively small intracellular domain responsible for interaction with the cytoskeleton and intracellular signaling pathways. To date, 18 alpha- and 8 beta-subunits have been identified, which associate selectively to form at least 24 different integrins. The unique combinations of alpha- and beta-subunits determine which ECM ligands are recognized by a cell. Binding of ECM ligands to integrins triggers interactions between several signaling molecules in close vicinity to the extracellular and cytoplasmic regions of the integrin receptor $(2,3)$. Integrin-mediated cell adhesion may affect the cell cycle kinetics, or may cause anchorage-dependent cell death or anoikis $(4,5)$. 
The alpha-subunit alphav can associate with various integrin beta-subunits. At present there are at least three distinct beta- subunits which associate with the alphav-subunit, namely, beta1, beta3, and beta5. The integrin alphavbeta3, also referred to as the vitronectin receptor, binds to a variety of extracellular matrix proteins, including vitronectin, fibrinogen, laminin, collagen, von Willebrand Factor and osteopontin through their exposed arginine-glycine-aspartic acid (RGD) amino acid sequence (6). Integrin alphavbeta 3 is expressed on a variety of cell types, including osteoclasts, vascular smooth muscle cells, and endothelial cells. In addition, alphavbeta 3 integrin is expressed on the cell membrane of various tumor cell types such as ovarian cancer, neuroblastoma, breast cancer, melanoma, and others. Furthermore, for growth beyond the size of 1-2 $\mathrm{mm}$ in diameter, tumors require the formation of new blood vessels. The formation of new blood vessels from pre-existing ones (angiogenesis) into an avascular tissue, is often triggered by an insufficient nutrient supply resulting in hypoxic cells (7). Binding of the hypoxia inducible factor (HIF-1alpha) to the hypoxia response element activates expression of vascular endothelial growth vector (VEGF), which plays a major role in angiogenesis. Integrin alphavbeta3 is minimally expressed on normal quiescent endothelial cells, but significantly upregulated on activated endothelial cells during angiogenesis (8). Alphavbeta3 integrin expressed on endothelial cells modulate cell migration and survival during angiogenesis, while alphavbeta 3 integrin expressed on carcinoma cells potentiate metastasis by facilitating invasion and movement across blood vessels.

Molecular imaging tracers for this integrin receptor could be used to noninvasively visualize alphavbeta3 expression in tumors. Noninvasive determination of alphavbeta3 expression potentially can be used to monitor treatment response to antiangiogenic drugs or even to select patients likely to respond to treatment with antiangiogenic drugs.

This review tries to give a brief overview on the currently used RGD-containing peptides as imaging probes for noninvasive visualization of alphavbeta3 expression using positron emission tomography (PET), single photon emission computed tomography (SPECT), magnetic resonance imaging (MRI), optical imaging (OI) and ultrasound (US).

\section{RGD-CONTAINING PEPTIDES AS IMAGING PROBES FOR ALPHAVBETA3 EXPRESSION}

\subsection{Nuclear imaging}

During the last decades, the application of radiolabeled peptides and proteins for diagnostic imaging and therapeutic treatment has rapidly gained importance in nuclear medicine.

Apart from planar imaging, SPECT and PET are the two main imaging modalities in nuclear medicine. SPECT imaging is much more widely available than PET imaging and the radionuclides used for SPECT are easier to prepare and usually have a longer half-life than those used for PET. Commonly used gamma emitters are: ${ }^{123} \mathrm{I}\left(\mathrm{E}_{\max }\right.$ $\left.529 \mathrm{keV}, \mathrm{t}_{1 / 2} 13.0 \mathrm{~h}\right),{ }^{111} \mathrm{In}\left(\mathrm{E}_{\max } 245 \mathrm{keV}, \mathrm{t}_{1 / 2} 67.2 \mathrm{~h}\right)$, and ${ }^{99 \mathrm{~m}} \mathrm{Tc}\left(\mathrm{E}_{\max } 141 \mathrm{keV}, \mathrm{t}_{1 / 2} 6.02 \mathrm{~h}\right)$. Compared to SPECT, PET has a higher spatial resolution and the possibility to more accurately quantitate in vivo the concentration of a tracer labeled with a positron emitting radionuclide for clinical application, such as ${ }^{18} \mathrm{~F}\left(\mathrm{E}_{\max } 635 \mathrm{keV}, \mathrm{t}_{1 / 2} 1.83 \mathrm{~h}\right)$, ${ }^{68} \mathrm{Ga}\left(\mathrm{E}_{\max } 1.90 \mathrm{MeV}, \mathrm{t}_{1 / 2} 68.1 \mathrm{~min}\right),{ }^{64} \mathrm{Cu}\left(657 \mathrm{keV}, \mathrm{t}_{1 / 2}\right.$ $12.7 \mathrm{~h})$, and for experimental use ${ }^{124} \mathrm{I}\left(\mathrm{E}_{\max } 2.13 \mathrm{MeV} ; 1.53\right.$ $\mathrm{MeV} ; 808 \mathrm{keV}, \mathrm{t}_{1 / 2} 4.18$ days).

PET is independent of the location depth of the reporter probe of interest and is able to detect picomolar concentrations of tracer (9). This high sensitivity of PET is only reached by optical imaging techniques, but not by MRI, computed tomography (CT) or ultrasound. In addition, compared to MRI and conventional optical imaging techniques, PET has the advantage that it can be quantitative. Though, with the introduction of fluorescence mediated tomography (FMT), quantitative measurements are also possible with OI techniques (10). However, the spatial resolution of PET and SPECT scanners used for clinical applications is lower compared to the spatial resolution of clinical MRI, CT and US scanners.

\subsubsection{Monomeric radiolabeled RGD peptides}

Integrin alphavbeta3 binds extracellular matrix proteins (e.g. vitronectin, fibrinogen, laminin, collagen) through exposed tripeptide arginine-glycine-aspartic acid (RGD) amino acid moieties (11). The potential of RGDcontaining peptides to serve as vehicles for targeting tumors with radionuclides has been investigated by several groups. It was found that the cyclic derivative cyclo(Arg-Gly-Asp-DPhe-Val) was a 100-fold better inhibitor of cell adhesion to vitronectin when compared to the linear variant, with an $\mathrm{IC}_{50}$ value in the lower nanomolar range $(12,13)$. It was shown that besides the essential RGD sequence, a hydrophobic amino acid in position 4 increases the affinity for alphavbeta3 (14). Based on this finding Haubner and coworkers designed five peptides that could be radioiodinated by introducing a tyrosine residue. Two of these peptides, cyclo(Arg-Gly-Asp-DTyr-Val) and cyclo(Arg-Gly-Asp-D-Phe-Tyr) (designated as $\mathrm{P} 1$ and $\mathrm{P} 4$, respectively) were studied in vivo (15). The biodistribution of the radioiodinated peptides was studied in nude mice with various tumors (M21 melanoma, MaCaF mammary carcinoma, and osteocarcoma). The peptides rapidly cleared from the blood; blood levels were lower than $1 \% \mathrm{ID} / \mathrm{g}$ as early as 10 min postinjection (p.i.). In mice with M21 melanoma, tumor uptake peaked at $10 \mathrm{~min}$ p.i. $(1.12 \pm 0.98 \% \mathrm{ID} / \mathrm{g})$ and decreased to $0.12 \pm 0.04 \% \mathrm{ID} / \mathrm{g}$ at $2 \mathrm{~h}$ p.i.. However, both peptides cleared via the hepatobiliary route and revealed relatively high hepatic uptake, especially at early time points $(\sim 5 \% \mathrm{ID} / \mathrm{g}, 1 \mathrm{~h}$ p.i.), which is unfavorable for patient studies. It has been demonstrated that introduction of sugar moieties resulted in reduced liver uptake and increased tumor accumulation of a variety of somatostatin receptor (SSTR)-antagonists $(16,17,18,19)$. Therefore, to improve the pharmacokinetics of the first generation radiohalogenated RGD peptides, sugar amino acids were introduced. A glucose-based sugar amino acid (SAA1) was conjugated to the epsilon-amino function of lysine in the 

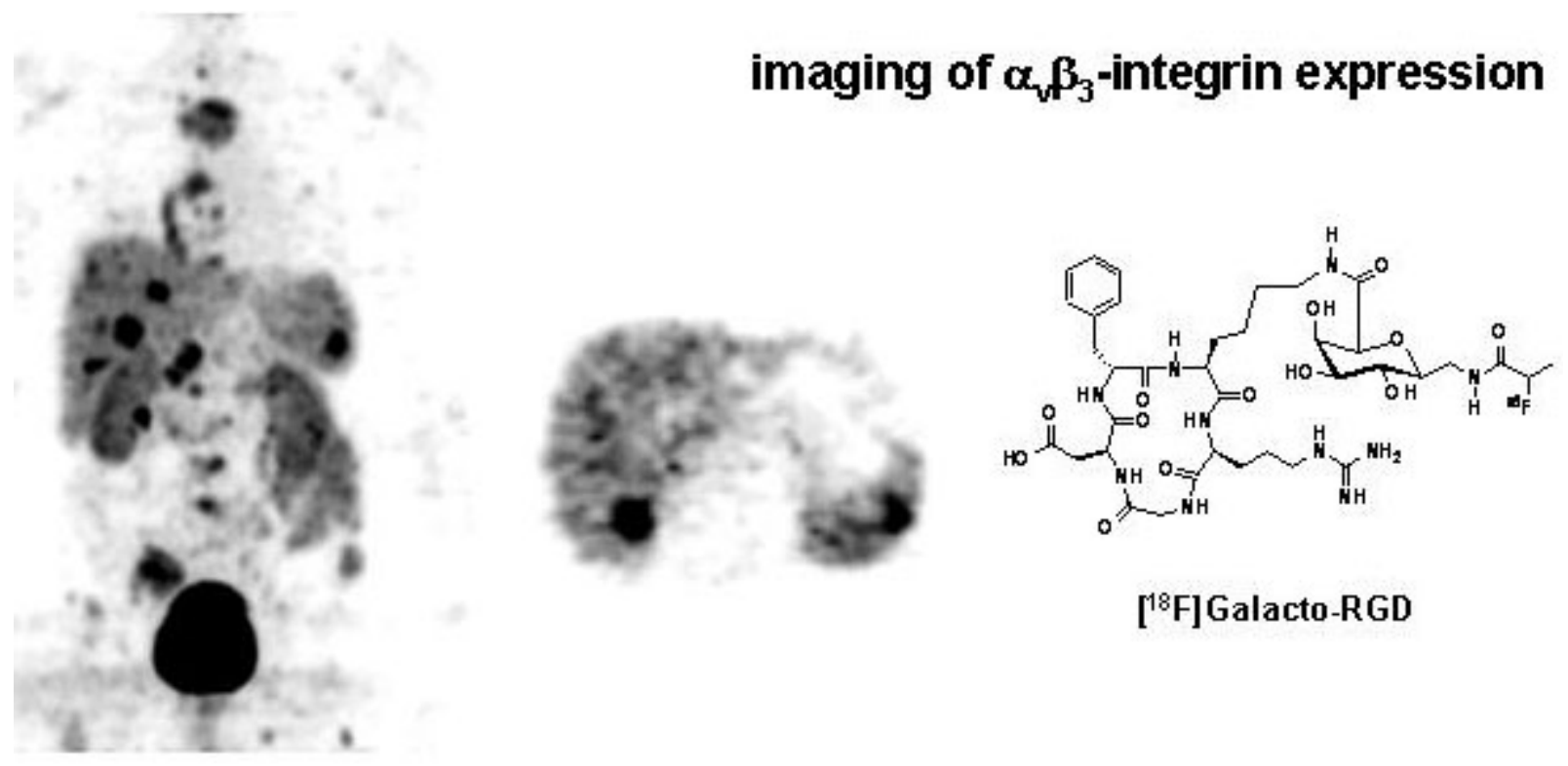

$\left[{ }^{18} \mathrm{~F}\right]$ Galacto-RGD

Figure 1. Structure of $\left[{ }^{18} \mathrm{~F}\right]$ Galacto-RGD and (left) $\left[{ }^{18} \mathrm{~F}\right]$ Galacto-RGD-PET (maximum intensity projection) of a patient with a carcinoid of the lung and multiple osseous, hepatic and lienal metastases; middle: corresponding axial view through liver and spleen.

pentapeptide. Compared to the noncarbohydrated radioiodinated $\mathrm{P} 4$, the resulting iodine labeled glucopeptide 3-[*I]iodo-D-Tyr4-cyclo(Arg-Gly-Asp-D-Tyr-Lys (SAA1)) (*I-Gluco-RGD) showed reduced activity accumulation in the liver, an initially increased activity concentration in the blood and an increased uptake and retention in the tumor (20). Based on these data a galactose-based sugar amino acid (SAA2) was conjugated with cyclo(Arg-Gly-Asp-DPhe-Lys) allowing prosthetic group labeling $(20,21)$. Due to the low lipophilicity of the small propionyl moiety, 4nitrophenyl-2- $\left[{ }^{18} \mathrm{~F}\right]$ fluoropropoinate $\left(\left[{ }^{18} \mathrm{~F}\right] \mathrm{NFP}\right)$ was used for ${ }^{18} \mathrm{~F}$-labeling. $\quad\left[{ }^{18} \mathrm{~F}\right]$ Galacto-RGD showed receptor specific visualization of alphavbeta3-positive tumors and thus was the first radiolabeled tracer for noninvasive determination of alphavbeta3 expression using PET (Figure $1)$.

Another strategy to improve pharmacokinetics of peptide-based tracers is conjugation of D-amino acids as hydrophilic moieties $(22,23)$. Two tetrapeptides containing a gamma-amino butyric acid and either three D-serine $\left(\mathrm{Ser}_{3}-\mathrm{RGD}\right)$ or three D-aspartic acids $\left(\mathrm{Asp}_{3}-\mathrm{RGD}\right)$ were synthesized, conjugated with Lys in the pentapeptide cyclo(Arg-Gly-Asp-D-Phe-Lys) and labeled with $\left[{ }^{18} \mathrm{~F}\right] \mathrm{NFP}$. These tracers were evaluated in vivo and $\left[{ }^{18} \mathrm{~F}\right] \mathrm{Asp}_{3}-\mathrm{RGD}$ showed lower tumor uptake compared to $\left[{ }^{18} \mathrm{~F}\right]$ GalactoRGD. However, due to faster elimination of $\left[{ }^{18} \mathrm{~F}\right] \mathrm{Asp}_{3^{-}}$ RGD, tumor-to-background ratios calculated from small animal PET images were comparable.

Conjugation to chelators like DTPA can also improve the pharmacokinetics of a tracer. Van Hagen et al. showed that the introduction of the DTPA-moiety in the pentapeptide cyclo(Arg-Gly-Asp-D-Tyr-Lys) via the epsilon-group of the lysine residue made the peptide more hydrophilic and facilitated renal clearance, while the non-DTPA-conjugated radioiodinated peptide predominantly cleared via the liver (24).

The hydrophilicity of peptides can also be enhanced by linking them to polyethylene glycol (PEG) chains, an approach called PEGylation. Chen and coworkers coupled PEG-moieties to RGD-containing peptides. Radioiodinated, ${ }^{18} \mathrm{~F}$ - and ${ }^{64} \mathrm{Cu}$-labeled derivatives were studied and demonstrated different effects of PEGylation on the pharmacokinetics, tumor uptake and retention of the RGD peptides. This could be due to the nature of the lead structure and the size of the PEG-moiety. For example, ${ }^{125} \mathrm{I}-\mathrm{RGD}-\mathrm{mPEG}$ (mPEG $\mathrm{MW}=2,000$ ) demonstrated higher activity concentration in liver and intestine compared to ${ }^{125}$ I-RGD. In addition, the PEGylated analog showed faster blood clearance, lower uptake, but improved tumor retention compared to ${ }^{125}$ I-RGD (25). In contrast, the PEGylated RGD peptide ${ }^{64} \mathrm{Cu}$-DOTA-PEGRGD (PEG MW = 3,400) revealed lower uptake in liver and intestine with no effect on tumor uptake and retention compared to ${ }^{64} \mathrm{Cu}$-DOTA-RGD (26). In an experimental comparison, $\left[{ }^{18} \mathrm{~F}\right]$ FB-PEG-RGD (PEG MW = 3,400) had significantly improved tumor retention relative to $\left[{ }^{18} \mathrm{~F}\right] \mathrm{FB}-$ RGD without compromising hepatic and renal clearance of activity (27).

Recently, a ${ }^{99 m}$ Tc-labeled RGD-containing peptide (NC100692) was evaluated in ischemic models and showed high uptake in areas of neovascularization with alphavbeta3 integrin overexpression (28). Binding of $\mathrm{NC} 100692$ has been confirmed to be localized on endothelial cells in the regions of angiogenesis (29). Subsequently, a clinical study was performed to provide an initial indication of the efficacy and safety of imaging 
malignant breast tumors using ${ }^{99 \mathrm{~m}} \mathrm{Tc}-\mathrm{NC} 100692$ (see "Translation into the clinic" section).

\subsubsection{RGD- multimers}

Multimers were developed to address the question of multimeric integrin binding by "poly-potent" ligands, and to initiate and target integrin clusters with noncarrier added (n.c.a.) radiopharmaceuticals $(30,31)$. The above mentioned alphavbeta3-selective peptides cyclo(RGDfK-) or cyclo(-RGDfE-) were linked to polyethylenglycol (PEG) amino acid and other spacers (32, $33,34)$. Monomeric units were bridged by lysine or a lysine tree to form dimeric, tetrameric and octameric RGDoligomers in a well-controlled, defined and characterized manner. The final ${ }^{18} \mathrm{~F}$-labeling step was carried out by oxime ligation, e.g. using $4-\left[{ }^{18} \mathrm{~F}\right]$ fluorobenzaldehyde. Comparison of the $\mathrm{IC}_{50}$ of cyclo(-RGDfK-) and cyclo(RGDfE-) containing monomers, dimers, tetramers and octamers for vitronection binding to alphavbeta3 revealed significantly increased affinity in the series monomer < dimer $<$ tetramer $<$ octamer. For example, the alphavbeta3affinities of the cyclo(-RGDfE-)-mono-, di- and tetramer containing heptaethylenglycol spacer units were increased by a factor of ten with each duplication of binding units $\left(\mathrm{IC}_{50}=20,3.0\right.$ and $0.2 \mathrm{nM}$, respectively for the mono-, diand tetramer). In contrast, the affinity of reference and control peptides carrying only one cyclo(-RGDfK-)- (or cyclo(-RGDfE-)-peptide, but otherwise non-binding cyclo(RADfK-)- or cyclo(-RADfE-)-sequences, respectively, was lower or similar to that of the corresponding monomers. Together, these experiments clearly demonstrate the "multimer effect" in vitro with similar molecular structures, which is independent of differences in charge, size or shape. These data were also confirmed in vivo in M21-melanoma-tumor-bearing-mice (32). Tumor uptake increased in the series monomer $<$ dimer $\approx$ tetramer, but due to lower activity accumulation of the tetramer in all other tissues compared to the dimer, tumor-to-organ-ratios were highest for the ${ }^{18} \mathrm{~F}$-labeled RGD-tetramer, leading to a significant improved imaging (Figure 2).

In a study by Chen and coworkers, the dimeric cyclic RGD peptide $\mathrm{E}(\mathrm{c}(\mathrm{RGDyK}))_{2}$ was labeled with ${ }^{18} \mathrm{~F}$ by using a prosthetic $4-\left[{ }^{18} \mathrm{~F}\right]$ fluorobenzoyl moiety to the amino group of the glutamate (35). The resulting $\left[{ }^{18} \mathrm{~F}\right] \mathrm{FB}$ $\mathrm{E}(\mathrm{c}(\mathrm{RGDyK}))_{2}$ was obtained with high specific activity (200-250 GBq/micromol at the end of synthesis). In mice with subcutaneously growing U87MG glioblastoma, the dimeric RGD peptide demonstrated significantly higher tumor uptake and prolonged tumor retention in comparison with its monomeric analog $\left[{ }^{18} \mathrm{~F}\right] \mathrm{FB}-\mathrm{c}(\mathrm{RGDyK})$. In addition, the dimeric peptide had a predominant renal excretion, whereas the monomeric analog was excreted preliminary through the biliary route.

The concept of multimerization has also been investigated with other RGD-ligand systems, such as ${ }^{64} \mathrm{Cu}$ and ${ }^{68} \mathrm{Ga}$-labeled peptide dimers, tetramers, and even octamers $(36,37,38,39,40,41,42)$. Compared with DOTA-RGD tetramer, DOTA-RGD octamer further increased the integrin affinity by a factor of $3\left(\mathrm{IC}_{50}=2.8 \pm\right.$ 0.4 and $1.1 \pm 0.2 \mathrm{nM}$, respectively). In vivo microPET imaging showed that $\left[{ }^{64} \mathrm{Cu}\right]$ DOTA-RGD octamer had slightly higher initial tumor uptake and longer tumor retention. However, the octamer exhibited significantly higher renal uptake in both subcutaneous U87MG xenografts and mammary adenocarcinoma-bearing c-neu oncomice compared with the tetramer, which resulted from integrin alphavbeta3 expression in kidneys, increased alphavbeta3-binding affinity and the presence of more positively charged amino acid residues (36). Overall, the multimerisation approach leads to increased binding affinity and tumor uptake as well as retention and can improve the pharmacokinetics of peptide-based tracers (for an overview see (43)).

\subsection{Magnetic resonance imaging}

MRI offers good depth penetration and its resolution is usually higher than that of clinical PET scanners although this depends on the exact protocol applied. MRI is widely used clinically to assess tumor growth and for response evaluation. Anatomical information can be coregistered with functional and molecular information within a single imaging method. A further advantage compared to radiotracer techniques is that MRI does not use ionizing radiation and generally is more widely available than PET. A major disadvantage of MRI compared to radiotracer techniques is its lower sensitivity for the detection of targeted agents. However, the problem of limited sensitivity might be overcome in the future by signal amplification strategies that generate higher target-to-background contrast (44).

Imaging of alphavbeta3 expression has been achieved by using $\mathrm{Gd}^{3+}$-containing paramagnetic liposomes with a diameter of $300-350 \mathrm{~nm}$ and the alphavbeta3 specific antibody LM609 as a ligand (45). Peptidomimetic integrin alphavbeta3 antagonist conjugated magnetic nanoparticles were also used for MRI in a $\mathrm{Vx}-2$ squamous cell carcinoma model with a common clinical MRI scanner at $1.5 \mathrm{~T}(46)$. The targeted nanoparticles increased the MR signal significantly in the periphery of the tumor at $2 \mathrm{~h}$ p.i.. Moreover, nude mice with human melanoma tumor xenografts were successfully imaged using alphavbeta3 integrin-targeted paramagnetic nanoparticles (47). The authors claimed that very small regions of about $30 \mathrm{~mm}^{3}$ of angiogenesis associated with melanoma tumor xenografts were visualized, which may enable characterizing and staging of early melanoma in a clinical setting.

However, $\mathrm{Gd}^{3+}$ for enhancing the $\mathrm{T} 1$ contrast can only be reliably detected at millimolar levels. Superparamagnetic iron oxide (SPIO) nanoparticles can be detected at a much lower concentration because of the high susceptibility induced by this particles which leads to a decrease of the signal in $\mathrm{T} 2$ and especially $\mathrm{T} 2 *$ weighted sequences ("negative contrast") (48). In a recent study, alphavbeta3 integrin-targeted ultrasmall SPIO (USPIO) nanoparticles were used for noninvasive differentiation of tumors with high and lower area fractions of alphavbeta3positive tumor vessels (49). After RGD-USPIO injection, T2*-weighted MRI identified the heterogeneous distribution of alphavbeta3-positive tumor vessels by an irregular signal intensity decrease, whereas the signal 

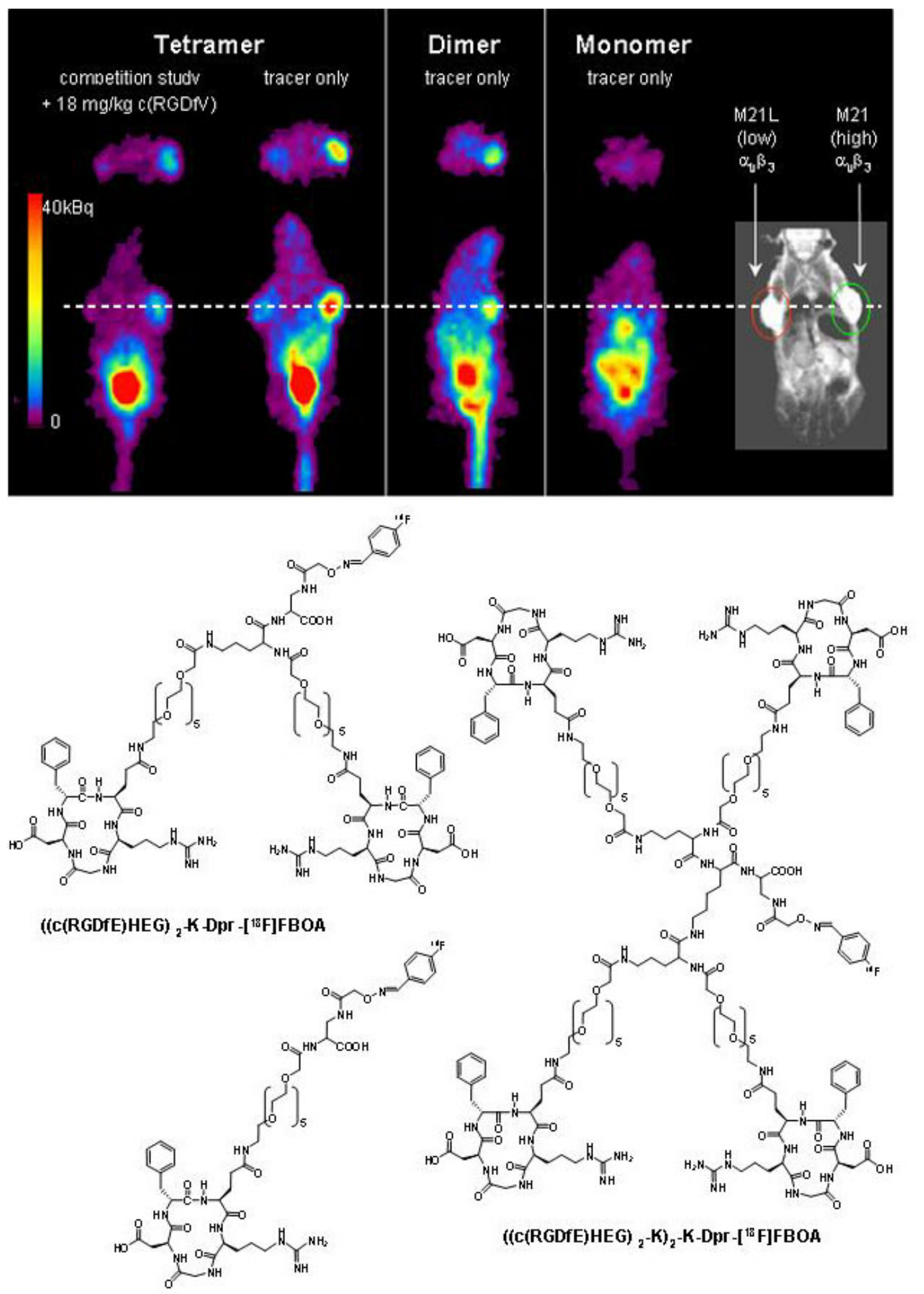

\section{C(RGDFE)HEG -Dpr - $\left[{ }^{18} \mathrm{~F}\right] \mathrm{FBOA}$}

Figure 2. Small animal PET images of M21- and M21L-melanoma tumor bearing nude mice 90 min p.i. of the $\left[{ }^{18} \mathrm{~F}\right] \mathrm{FBOA}-$ labeled (c(RGDfE)HEG-Dpr- $\left.\left[{ }^{18} \mathrm{~F}\right] \mathrm{FBOA}\right)$ (monomer), ((c(RGDfE)HEG)2-K-Dpr- $\left.\left[{ }^{18} \mathrm{~F}\right] \mathrm{FBOA}\right)(\mathrm{dimer})$ and (((c (RGDfE)HEG)2$\left.\mathrm{K}) 2-\mathrm{K}-\mathrm{Dpr}-\left[{ }^{18} \mathrm{~F}\right] \mathrm{FBOA}\right)$ (tetramer) with corresponding structures. Specificity of tumor uptake of the tetramer is demonstrated in a competition study after coinjection of $18 \mathrm{mg} / \mathrm{kg}$ c(RGDfV). The MRI-image on the right indicates the position of the respective tumor with high (M21) and low (M21L) alphavbeta3-expression (HEG = 20-amino-3,6,9,12,15,18-hexaoxaeicosanoic acid). 
intensity decreased more homogeneously in the control tumor with predominantly small and uniformly distributed vessels.

Imaging based on $\mathrm{T} 2 \mathrm{w}$ sequences is also possible with iron-oxide based dual modality nanoparticles, like RGD-CLIO-Cy5.5, which allow both for MRI and fluorescence imaging (50). This approach combines the high sensitivity of optical imaging with the high spatial resolution of MRI. Our lab recently developed a bifunctional iron oxide (IO) nanoparticle probe for PET and MR imaging of tumor integrin alphavbeta3 expression. With a core size of 5-7 nm, Poly (aspartic acid) (PASP)coated IO nanoparticles (PASP-IO) were coupled to cyclic RGD peptides and macrocyclic DOTA chelators to get a bifunctional probe - DOTA-IO-RGD conjugates. The probe bound specifically to integrin alphavbeta3 in vitro. Both microPET and T2-weighted MR imaging showed integrinspecific delivery of conjugated RGD-PASP-IO nanoparticles and prominent reticuloendothelial system (RES) uptake. This bifunctional imaging approach may allow for earlier tumor detection with a high degree of accuracy and provide further insight into the molecular mechanisms of cancer.

\subsection{Ultrasound}

Ultrasound has a high spatial resolution $(50-500$ micrometer) yet it also has disadvantages such as the relatively poor depth penetration (usually a few centimeters depending on the frequency used) and limited sensitivity. For integrin alphavbeta3 targeted contrast enhanced ultrasound ("CEU"), the shell surface of microbubbles has been conjugated with echistatin, a peptide derived from the venom of the viper Echis carinatus which bears the RGD motif (51). Using gray-scale pulse-inversion techniques, tumor-blood volume determined by CEU increased by approximately $35 \%$ from day 14 to day 28 , whereas microvascular blood velocity decreased, especially at the central portions of the tumors (52). In another study, antihuman integrin alphavbeta3 antibody conjugated microbubbles and cyclic RGD peptide conjugated microbubbles have been prepared for in vitro ultrasonic analysis. Specific adhesion of these contrast agents to alphavbeta3-expressing cell monolayers was achieved in vitro and acoustic studies illustrated a backscatter amplitude increase from monolayers exposed to the targeted contrast agents of up to 13 -fold $(22 \mathrm{~dB})$ relative to enhancement due to control bubbles (53). Tartis et al. constructed drug delivery vehicles, referred to as acoustically active lipospheres (AALs), which are microbubbles surrounded by a shell of oil and lipid (54). In a region limited to the focal area of ultrasound application, circulating AALs are deflected by radiation force to a vessel wall and can subsequently be fragmented. RGD peptide conjugated AAL shell showed an increase in in vitro binding by 26.5 -fold over nontargeted agents. Toxicity assays demonstrate that paclitaxel-containing AALs exert a greater antiproliferative effect after insonation than free paclitaxel at an equivalent concentration. The combination of ultrasound and molecular targeting successfully delivered a model drug to the endothelium and interstitium of chorioallantoic membrane vasculature in vivo (54).

\subsection{Optical imaging of alphavbeta3 expression}

Optical imaging and in particular, near-infrared fluorescence (NIRF) imaging, which makes use of photons emitted in the near-infrared and far red range, has the advantage of being relatively inexpensive, highly sensitive and noninvasive. Due to its limited depth penetration, it is mainly applied in preclinical animal studies and in superficial tissues or in combination with endoscopy. Nearinfrared light penetrates tissue sufficiently well to allow one to obtain low-resolution images of tissues to a depth of a few centimetres, and the regional concentration of hemoglobin and oxygen saturation can be calculated from the absorption of hemoglobin and deoxyhemoglobin. Limitations of OI include its limited depth penetration, the effects of blood absorption and autofluorescence. Moreover, conventional optical imaging does not allow for quantitative measurements. However, this has changed with the introduction of fluorescence mediated tomography (FMT), which allows for quantitative measurements of fluorochrome concentrations at different tissue depths (10). Fluorescence imaging has been used for retinal angiography, cardiovascular surgery, and gastrointestinal endoscopy using either indocyanine green, a clinically available contrast agent that fluoresces at near-infrared wavelengths, or autofluorescence. Indocyanine green has also been used to image angiogenic vasculature in breast tumors $(55,56)$. In addition, a vast array of sensitive molecular imaging agents associated with angiogenesis specific targets has been developed for OI techniques, such as agents sensitive for matrix metalloproteases or alphavbeta3 (57). The simplest technique is the use of cyanine dyes like Cy5.5 coupled to RGD peptides. This approach has been successfully used for imaging of U87MG tumor xenografts in mice using NIRF (58). Analogous to the radiotracer approach, multimeric compounds like Cy5-RAFT-c (-RGDfK-)4 demonstrated increased binding affinity compared to the monomeric compound (59). Finally, the use of FMT allows for quantification of fluorochrome concentrations in living subjects, therefore potentially allowing for quantification of alphavbeta3 expression by RGD-specific cyanine dyes. The principle of FMT using Cy5.5-RGD has recently been successfully demonstrated by the group of Bremer et al. (60). This approach is therefore well suited for high throughput studies and a potential alternative to radiotracer studies in the preclinical setting.

Another strategy is to use quantum dots (QD) for OI. QDs are inorganic fluorescent semiconductor nanoparticles with many desirable optical properties for imaging applications, such as high quantum yields, high molar extinction coefficients, strong resistance to photobleaching and chemical degradation, continuous absorption spectra spanning the UV to NIR range and narrow emission spectra, typically $20-30 \mathrm{~nm}$ full width at half maximum $(61,62,63)$. Specific targeting can be achieved by attaching targeting ligands to the QD surface. However, significant limitations for in vivo imaging are the 

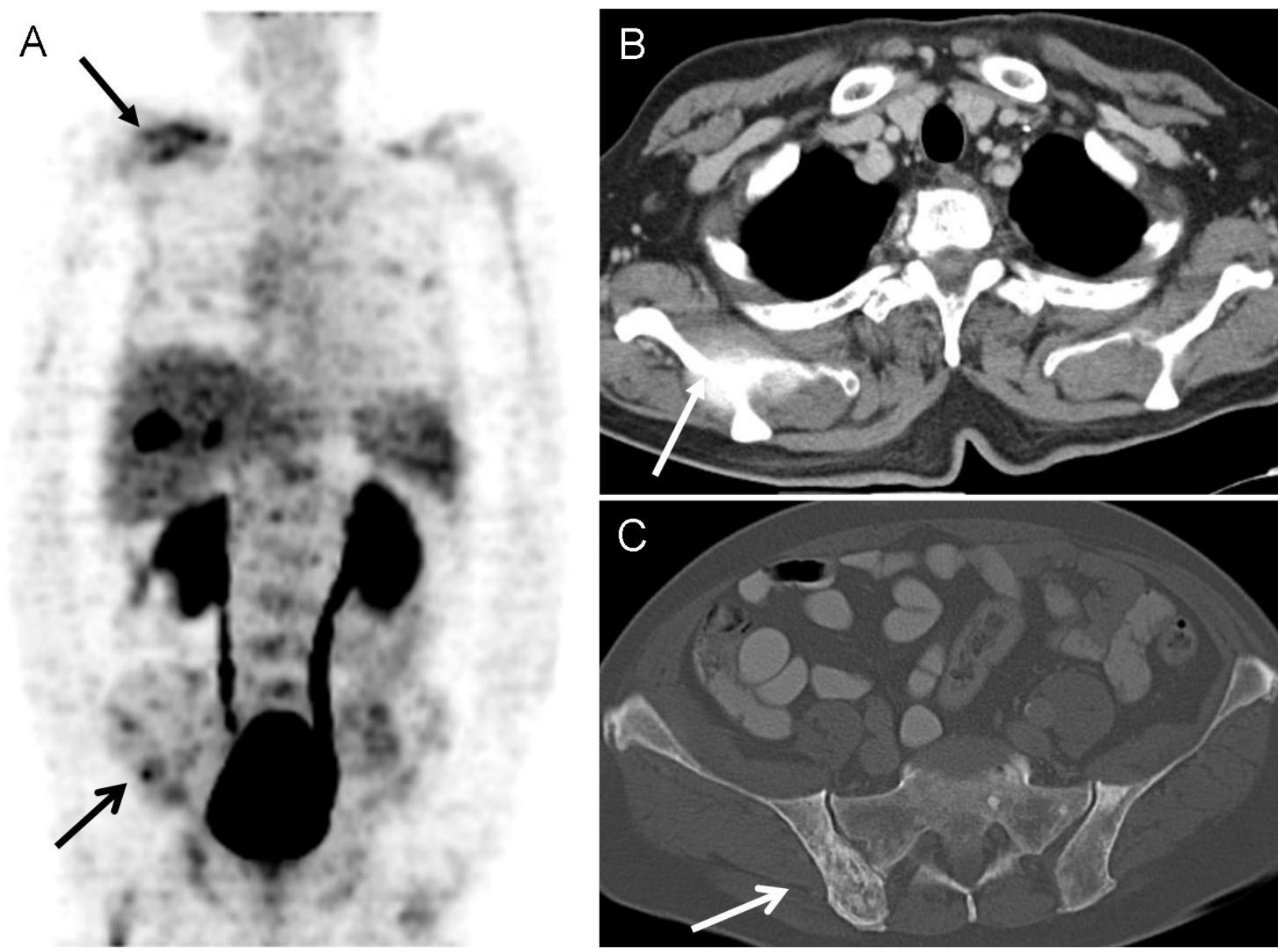

Figure 3. Patient with diffuse osseous metastases from prostate carcinoma. The maximum intensity projection (MIP) of the $\left[{ }^{18} \mathrm{~F}\right]$ Galacto-RGD PET scan shows focal and diffuse tracer uptake in the whole skeleton, most intense in the right scapula (arrow, closed tip) and in the pelvis (arrow, open tip). Note, the tracer accumulation in the urogenital tract due to predominantly renal tracer elimination, and to a lesser extent in the gallbladder, liver and spleen. Only low tracer uptake is notable in the residual organs and background. The corresponding CT scan shows the osseous metastasis in the scapula (B) and in the pelvis (C).

relatively large overall size over $20 \mathrm{~nm}$ and the short circulation half-life of QD conjugates. NIRF imaging of integrin alphavbeta3 on the tumor vasculature has been reported using RGD peptide-conjugated QDs (64). RGD peptides were conjugated to QD705 (emission maximum at $705 \mathrm{~nm}$ ) and QD705-RGD exhibited in cell culture and ex vivo high affinity integrin alphavbeta 3 specific binding. Tumor contrast was observed as early as 20 minutes after QD705-RGD injection and the fluorescence intensity in subcutaneous U87MG tumors reached maximum at $6 \mathrm{~h}$ p.i.. Spectral imaging techniques were used to better interpret the NIRF imaging result since significant background signal is usually observed for in vivo NIRF imaging because of tissue autofluorescence $(65,66)$. The large size of QD705-RGD with $\sim 20 \mathrm{~nm}$ in diameter prevented extravasation, which was confirmed by ex vivo immunofluorescence staining. Thus QD705-RGD mainly targeted integrin alphavbeta3 on the tumor vasculature instead of the tumor cells. The major limitations concerning a clinical translation of QDs are the short half-life, potential toxicity, and lack of quantification (61). However, with the development of smaller, less toxic and multifunctional QDs, QD-based probes might be optimized with acceptable toxicity for clinical translation in the future $(67,68,69)$.

\section{TRANSLATION INTO THE CLINIC}

Up to now, the one of the few approaches of imaging alphavbeta3 expression which has made the transition from bench to bedside is the radiotracer approach. $\left[{ }^{18} \mathrm{~F}\right]$ Galacto-RGD was one of the first substances applied in patients and could successfully image alphavbeta3 expression in human tumors with good tumorto-background ratios (70) (Figure 3). In all patients, rapid, predominantly renal tracer elimination was observed, resulting in low background activity in most regions of the body. The metabolic stability analyzed from blood samples was $>96 \%$ intact tracer 120 min after tracer injection. Further biodistribution and dosimetry studies confirmed rapid clearance of $\left[{ }^{18} \mathrm{~F}\right]$ Galacto-RGD from the blood pool and primarily renal excretion (71). The calculated effective dose found was approximately 19 microSv/MBq, which is 

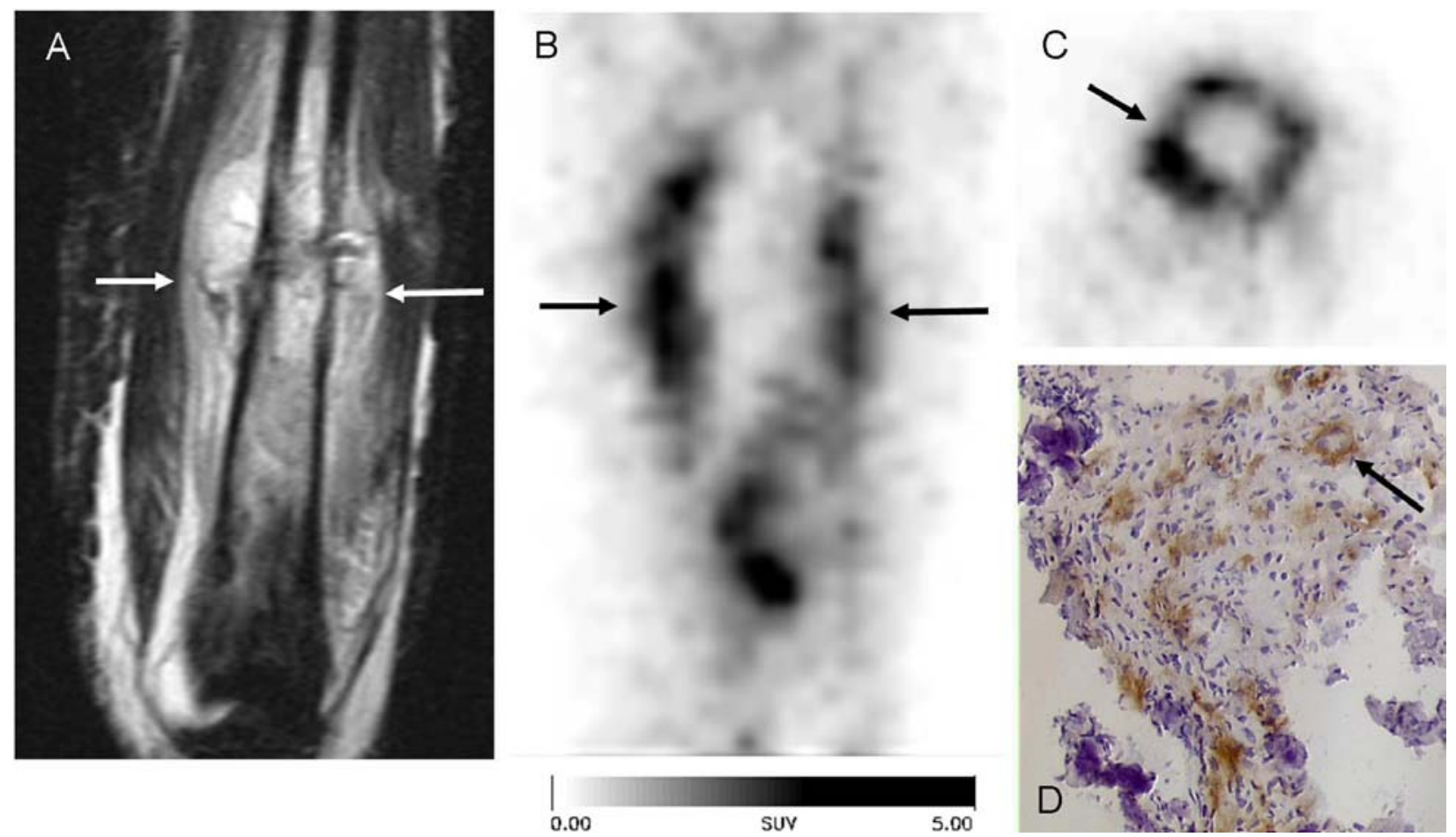

Figure 4. Osteosarcoma of the femur. The tumor can be clearly seen in the coronal T2-weighted MRI (A) as a bright mass in the diaphysis of the femur with a large soft tissue mass adjacent to the bone (arrows). The corresponding coronal view (B) and axial view (C) of the $\left[{ }^{18} \mathrm{~F}\right]$ Galacto-RGD PET scan show intense tracer uptake in most parts of the tumor. Immunohistochemistry of alphavbeta3 expression shows positive staining of the tumor specimen (D).

very similar to an $\left[{ }^{18} \mathrm{~F}\right] \mathrm{FDG}$ scan (72). Dynamic studies with tracer kinetic modelling were used to assess distribution volume $\left(\mathrm{D}_{\mathrm{v}}\right)$ values, which are supposed to reflect the receptor concentration in the tissue. These were on average four times higher for tumor tissue than for muscle tissue, suggesting specific tracer binding (73).

In the lesions, tracer uptake revealed great interindividual heterogeneity. Moreover, there was also a great intraindividual heterogeneity of tracer uptake in different tumor sites in one patient which suggests great diversity of alphavbeta 3 expression. This also emphasizes the potential value of noninvasive techniques for selection of patients entering clinical trials with alphavbeta3-targeted therapies, who could be stratified according to individual integrin expression levels. For this purpose, a quantification of integrin expression would be necessary. Therefore we evaluated, if $\left[{ }^{18} \mathrm{~F}\right]$ Galacto-RGD uptake correlates with alphavbeta3 expression. 19 patients with solid tumors were examined with PET using $\left[{ }^{18} \mathrm{~F}\right]$ Galacto-RGD before surgical removal of the lesions (74). Tumor tissue sections from representative areas with low as well as high SUVs were collected and immunohistochemistry was performed. Intensity of staining and the microvessel density of alphavbeta3-positive vessels were determined and correlated with SUV and tumor-to-blood ratios (Figure 4). SUVs and tumor-to-blood ratios were found to correlate significantly with the intensity of immunohistochemical staining as well as with the microvessel density. Moreover, immunohistochemistry confirmed lack of alphavbeta3 expression in normal tissue and in the two tumors without tracer uptake. Thus, we could show that molecular imaging of alphavbeta3 expression with $\left[{ }^{18} \mathrm{~F}\right]$ Galacto-RGD in humans correlates with alphavbeta3 expression as determined by immunohistochemistry. We are now systematically examining different tumor entities with respect to their alphavbeta3 expression patterns as shown by $\left[{ }^{18} \mathrm{~F}\right]$ Galacto-RGD PET. In squamous cell carcinoma of the head and neck (SCCHN) we could demonstrate good tumor-to-background ratios with $\left[{ }^{18} \mathrm{~F}\right]$ Galacto-RGD PET, but again also a widely varying intensity of tracer uptake. Immunohistochemistry demonstrated predominantly vascular alphavbeta3 expression, thus in SCCHN, $\left[{ }^{18} \mathrm{~F}\right]$ Galacto-RGD PET might be used as a surrogate parameter of angiogenesis (75).

We have also compared the tracer uptake of $\left[{ }^{18} \mathrm{~F}\right] \mathrm{FDG}$ and $\left[{ }^{18} \mathrm{~F}\right]$ Galacto-RGD in patients with non small cell lung cancer (NSCLC, $\mathrm{n}=10$ ) and various other tumors $(n=8)$ because in case of a close correlation of the two tracers, there would probably be no need for a specific tracer like $\left[{ }^{18} \mathrm{~F}\right]$ Galacto-RGD. However, no correlation between the two tracers concerning all lesions $(r=0.157)$ could be demonstrated. For the subgroup of $\left[{ }^{18} \mathrm{~F}\right] \mathrm{FDG}$-avid lesions and lesions in patients with NSCLC, there was a slight trend towards a higher $\left[{ }^{18} \mathrm{~F}\right]$ Galacto-RGD uptake in more $\left[{ }^{18} \mathrm{~F}\right]$ FDG-avid lesions $(\mathrm{r}=0.337)$. However, the correlation coefficient was very low. Our results suggests that alphavbeta3 expression and glucose metabolism are not closely correlated in tumor lesions and that 
consequently $\left[{ }^{18} \mathrm{~F}\right] \mathrm{FDG}$ cannot provide similar information as $\left[{ }^{18} \mathrm{~F}\right]$ Galacto-RGD (76).

As mentioned before, variation in tracer design is supposed to further improve the performance of alphavbeta3 imaging, e.g. using multimeric RGD peptides. Recently, the SPECT tracer $\left[{ }^{99 \mathrm{~m}} \mathrm{Tc}\right] \mathrm{NC} 100692$ was introduced for imaging alphavbeta 3 expression in patients with breast cancer. 19 of 22 tumors could be detected with this agent, which was safe and well tolerated by the patients (77). This is in accordance with results obtained in breast cancer patients using $\left[{ }^{18} \mathrm{~F}\right]$ Galacto-RGD PET, which showed heterogeneous uptake in primary as well as metastatic breast cancer (78). It can be assumed, that agents for alphavbeta3 imaging will be commercially available in the near future enabling a more widely assessment of alphavbeta3-targeting probes in larger cohorts of patients.

\section{CONCLUSIONS}

Effective molecular imaging is not only depending on the continuing improvement in imaging equipment and technology, but is also strongly dependent on the availability of powerful probes with optimal in vivo biodistribution and imaging characteristics.

In the last few years, significant progress has been made on the development of tracers for the visualization of alphavbeta3-integrin expression by different modalities (e.g. nuclear imaging, magnetic resonance imaging, computed tomography, ultrasound) and a considerable variety of imaging techniques is now available on the preclinical level. Up to now only $\left[{ }^{18} \mathrm{~F}\right]$ Galacto-RGD and $\left[{ }^{99 \mathrm{~m}} \mathrm{Tc}\right] \mathrm{NC} 100692$ have been transferred from bench to bedside and are currently assessed in clinical studies. These studies will provide new insight into the exact role of alphavbeta3 in the context of angiogenesis and tumor growth, and, together with ex vivo studies, will elucidate more information on the correlation of alphavbeta3 expression and other potential targets for antiangiogenic therapies, like MMP- and VEGF-/VEGF-receptor targeted drugs. In addition, newly developed high affinity binders to other integrin targets, such as alpha5beta1, and combinations of different imaging modalities might open further and eventually more selective routes to image angiogenic processes in animal models and patients with higher sensitivity and resolution.

\section{REFERENCES}

1. John Tamkun, Douglas DeSimone, Deborah Fonda, Ramila Patel, Clayton Buck, Alan Horwitz and Richard Hynes: Structure of integrin, a glycoprotein involved in the transmembrane linkage between fibronectin and actin. Cell 46, 271-281 (1986)

2. A.E. Aplin, A. Howe, S.K. Alahari and R.L. Juliano: Signal transduction and signal modulation by cell adhesion receptors: the role of integrins, cadherins, immunoglobulincell adhesion molecules, and selectins. Pharmacol Rev 50, 197-263 (1998)
3. Simone Schoenwaelder and Keith Burridge: Bidirectional signaling between the cytoskeleton and integrins. Curr Opin Cell Biol 11, 274-286 (1999)

4. Steven Frisch and Erkki Ruoslahti: Integrins and anoikis. Curr Opin Cell Biol 9, 701-706 (1997)

5. Alan Howe, Andrew Aplin, Suresh Alahari and RL. Juliano: Integrin signaling and cell growth control. Curr Opin Cell Biol 10, 220-231 (1998)

6. F. Patrick Ross, Jean Chappel, Jose Alvarez, Diane Sander, William Butler, Mary Farach-Carson, Keith Mintz, Pamela Gehron Robey, Steven Teitelbaum and David Cheresh: Interactions between the bone matrix proteins osteopontin and bone sialoprotein and the osteoclast integrin alphavbeta3 potentiate bone resorption. J Biol Chem 268, 9901-9907 (1993)

7. Peter Carmeliet and Rakesh K. Jain: Angiogenesis in cancer and other diseases. Nature 407, 249-257 (2000)

8. Peter Brooks, Richard Clark and David Cheresh: Requirement of vascular integrin alphavbeta3 for angiogenesis. Science 264, 569-571 (1994)

9. Wolfgang Weber: Positron emission tomography as an imaging biomarker. J Clin Oncol 24, 3282-3292 (2006)

10. Farouc Jaffer and Ralph Weissleder: Molecular Imaging in the Clinical Arena. JAMA 293, 855-862 (2005)

11. David Cheresh: Integrins: Structure, function and biological properties. Adv Mol Cell Biol 6, 225-252 (1993)

12. Monique Aumailley, Marion Gurrath, Gerhard Müller, Juan Calvete, Rupert Timpl and Horst Kessler: Arg-Gly-Asp constrained within cyclic pentapeptides. Strong and selective inhibitors of cell adhesion to vitronectin and laminin fragment P1. FEBS Lett 291, 5054 (1991)

13. Marion Gurrath, Gerhard Müller, Horst Kessler, Monique Aumailley and Rupert Timpl: Conformation/activity studies of rationally designed potent anti-adhesive RGD peptides. Eur J Biochem 210, 911-921 (1992)

14. Roland Haubner, Rainer Gratias, Beate Diefenbach, Simon Goodman, Alfred Jonczyk and Horst Kessler: Structural and functional aspects of RGD-containing cyclic pentapeptides as highly potent and selective integrin alphavbeta3 antagonists. J Am Chem Soc 118, 7461-7472 (1996)

15. Roland Haubner, Hans-Jürgen Wester, Ute Reuning, Reingard Senekowitsch-Schmidtke, Beate Diefenbach, Horst Kessler, Gerhard Stöcklin and Markus Schwaiger: Radiolabeled alphavbeta3 integrin antagonists: A new 
class of tracers for tumor targeting. J Nucl Med 40, 1061-1071 (1999)

16. M. Leisner, H. Kessler, M. Schwaiger, H.J. Wester: Synthesis of Na-D-Phe1-Amadori derivatives of Tyr3octreotide: Precursor for 123I/18F-labelled SSTR-binding SPECT/PET tracers with improved biodistribution. $J$ Label Compd Radiopharm 42, S549-S551 (1999)

17. Margret Schottelius, Hans-Jürgen Wester, Jean Claude Reubi, Reingard Senekowitsch-Schmidtke and Markus Schwaiger: Improvement of pharmacokinetics of radioiodinated Tyr (3)-octreotide by conjugation with carbohydrates. Bioconjugate Chem 13, 1021-1030 (2002)

18. Hans-Jürgen Wester, Margret Schottelius, Klemens Scheidhauer, Günther Meisetschläger, Michael Herz, Friederike Rau, Jean Claude Reubi and Markus Schwaiger: PET imaging of somatostatin receptors: design, synthesis and preclinical evaluation of a novel 18F-labelled, carbohydrated analogue of octreotide. Eur J Nucl Med Mol Imaging 30, 117-122 (2003)

19. Hans-Jürgen Wester, Margret Schottelius, Klemens Scheidhauer, Jean-Claude Reubi, Ingo Wolf and Markus Schwaiger: Comparison of radioiodinated TOC, TOCA and Mtr-TOCA: the effect of carbohydration on the pharmacokinetics. Eur J Nucl Med Mol Imaging 29, 28-38 (2002)

20. Roland Haubner, Hans-Jürgen Wester, Fred Burkhart, Reingard Senekowitsch-Schmidtke, Wolfgang Weber, Simon Goodman, Horst Kessler and Markus Schwaiger: Glycosylated RGD-containing peptides: tracer for tumor targeting and angiogenesis imaging with improved biokinetics. J Nucl Med 42, 326-336 (2001)

21. Roland Haubner, Bertrand Kuhnast, Christian Mang, Wolfgang Weber, Horst Kessler, Hans-Jürgen Wester and Markus Schwaiger: [18F]Galacto-RGD: Synthesis, radiolabeling, metabolic stability, and radiation dose estimates. Bioconjugate Chem 15, 61-69 (2004)

22. R. Haubner, B. Kuhnast, H. J. Wester, W. A. Weber, R. Huber, R. Senekowitsch-Schmidtke, S. I. Ziegler, S. L. Goodman, H. Kessler and M. Schwaiger: [18F]-RGDpeptides conjugated with hydrophilic tetrapeptides for the noninvasive determination of the alpha (v)beta3 integrin. $J$ Nucl Med 43, 89P (2002)

23. Roland Haubner: Alphavbeta3-integrin imaging: a new approach to characterise angiogenesis? Eur J Nucl Med Mol Imaging 33, 54-63 (2006)

24. P.M. van Hagen, W.A. Breeman, H.F. Bernard, M. Schaar, C.M. Mooij, A. Srinivasan, M.A. Schmidt, E.P. Krenning and M. de Jong: Evaluation of a radiolabelled cyclic DTPA-RGD analogue for tumour imaging and radionuclide therapy. Int J Cancer 90, 186-198 (2000)

25. Xiaoyuan Chen, Ryan Park, Anthony Shahinian, James Bading and Peter Conti: Pharmacokinetics and tumor retention of 125I-labeled RGD peptide are improved by PEGylation. Nucl Med Biol 31, 11-19 (2004)

26. Xiaoyuan Chen, Yingping Hou, Michel Tohme, Ryan Park, Vazgen Khankaldyyan, Ignacio Gonzales-Gomez, James Bading, Walter Laug and Peter Conti:_Pegylated Arg-Gly-Asp peptide: $64 \mathrm{Cu}$ labeling and PET imaging of brain tumor alphavbeta3-integrin expression. $\mathrm{J} \mathrm{Nucl} \mathrm{Med}$ $45,1776-1783$ (2004)

27. Xiaoyuan Chen, Ryan Park, Yingping Hou, Vazgen Khankaldyyan, Ignacio Gonzales-Gomez, Michel Tohme, James Bading, Walter Laug and Peter Conti: MicroPET imaging of brain tumor angiogenesis with 18F-labeled PEGylated RGD peptide. Eur J Nucl Med Mol Imaging 31, 1081-1089 (2004)

28. Merry Lindsey, G. Patricia Escobar, Lawrence Dobrucki, Danielle Goshorn, Shenikqua Bouges, Joseph Mingoia, David McClister, Jr., Haili Su, Joseph Gannon, Catherine MacGillivray, Richard Lee, Albert Sinusas, and Francis Spinale: Matrix metalloproteinase- 9 gene deletion facilitates angiogenesis after myocardial infarction. $\mathrm{Am} \mathrm{J}$ Physiol Heart Circ Physiol 290, H232-H239 (2006)

29. Jing Hua, Lawrence Dobrucki, Mehran Sadeghi, Jiasheng Zhang, Brian Bourke, Patti Cavaliere, James Song, Conroy Chow, Neda Jahanshad, Niels van Royen, Ivo Buschmann, Joseph Madri, Marivi Mendizabal, Albert Sinusas: Noninvasive imaging of angiogenesis with a 99mTc-labeled peptide targeted at alphavbeta3 integrin after murine hindlimb ischemia. Circulation 111, 32553260 (2005)

30. M. Bock, R. Haubner, R. Senekowitsch-Schmidtke, H. Kessler, G. Stöcklin, M. Schwaiger and H.J. Wester: Homo- and heteromultimeric ligand systems as tracers for tumorimaging und endoradiotherapy. Nuclear Med 38, A44 (abstract) (1999)

31. Hans-Jürgen Wester and Horst Kessler: Molecular targeting with peptides or peptide-polymer-conjugates: just a question of size? J Nucl Med 46, 1940-1945 (2005)

32. Thorsten Poethko, Margret Schottelius, Georgette Thumshirn, Michael Herz, Roland Haubner, Gjermund Henriksen, Horst Kessler, Markus Schwaiger and HansJürgen Wester: Chemoselective pre-conjugate radiohalogenation of unprotected mono- and multimeric peptides via oxime formation. Radiochim Acta 92, 317-328 (2004)

33. Thorsten Poethko, Margret Schottelius, Georgette Thumshirn, Ulrich Hersel, Michael Herz, Gjermund Henriksen, Horst Kessler, Markus Schwaiger and HansJürgen Wester: Two-step methodology for high-yield routine radiohalogenation of peptides: (18)F-labeled RGD and octreotide analogs. J Nucl Med 45, 892-902 (2004)

34. Georgette Thumshirn, Ulrich Hersel, Simon Goodman and Horst Kessler: Multimeric cyclic RGD peptides as potential tools for tumor targeting: solid-phase peptide 
synthesis and chemoselective oxime ligation. Chemistry 9 , 2717-2725 (2003)

35. Xiaoyuan Chen, Michel Tohme, Ryan Park, Yingping Hou, James Bading and Peter Conti: Micro-PET imaging of alphavbeta3-integrin expression with $18 \mathrm{~F}$-labeled dimeric RGD peptide. Mol Imaging 3, 96-104 (2004)

36. Zi-bo Li, Weibo Cai, Qizhen Cao, Kai Chen, Zhanhong $\mathrm{Wu}$, Lina $\mathrm{He}$ and Xiaoyuan Chen: (64)Cu-labeled tetrameric and octameric RGD peptides for small-animal PET of tumor alpha (v)beta (3) integrin expression. $J$ Nucl Med 48, 1162-1171 (2007)

37. Shuang Liu, Wen-Yuan Hsieh, Young Jiang, YoungSeung Kim, Subramanya Sreerama, Xiaoyuan Chen, Bing Jia and Fan Wang: Evaluation of a $(99 \mathrm{~m}) \mathrm{Tc}$-labeled cyclic RGD tetramer for noninvasive imaging integrin alpha (v)beta3-positive breast cancer. Bioconjugate Chem 18, 438-446 (2007)

38. Zhen Cheng, Yun Wu, Zhengming Xiong, Sanjiv Sam Gambhir and Xiaoyuan Chen: Near-infrared fluorescent RGD peptides for optical imaging of integrin alphavbeta3 expression in living mice. Bioconjugate Chem 16, 1433$1441(2005)$

39. Yun Wu, Xianzhong Zhang, Zhengming Xiong, Zhen Cheng, Darrell Fisher, Shuang Liu, Sanjiv Gambhir and Xiaoyuan Chen: microPET imaging of glioma integrin \{alpha\}v\{beta\}3 expression using (64)Cu-labeled tetrameric RGD peptide. J Nucl Med 46, 1707-1718 (2005)

40. Ingrid Dijkgraaf, Anneloes Rijnders, Annemieke Soede, Annemarie Dechesne, G. Wilma van Esse, Arwin Brouwer, Frans Corstens, Otto Boerman, Dirk Rijkers and Rob Liskamp: Synthesis of DOTA-conjugated multivalent cyclic-RGD peptide dendrimers via 1,3-dipolar cycloaddition and their biological evaluation: implications for tumor targeting and tumor imaging purposes. Org Biomol Chem 5, 935-944 (2007)

41. Ingrid Dijkgraaf, John Kruijtzer, Shuang Liu, Annemieke Soede, Wim Oyen, Frans Corstens, Rob Liskamp and Otto Boerman: Improved targeting of the alpha (v)beta (3) integrin by multimerisation of RGD peptides. Eur J Nucl Med Mol Imaging 34, 267-273 (2007)

42. Marcel Janssen, Wim Oyen, Leon Massuger, Cathelijne Frielink, Ingrid Dijkgraaf, D. Scott Edwards, Milind Radjopadhye, Frans Corstens and Otto Boerman: Comparison of a monomeric and dimeric radiolabeled RGD-peptide for tumor targeting. Cancer Biother Radiopharm 17, 641-646 (2002)

43. Shuang Liu: Radiolabeled multimeric cyclic RGD peptides as integrin alphavbeta 3 targeted radiotracers for tumor imaging. Mol Pharm_3, 472-487 (2006)

44. Farouc Jaffer and Ralph Weissleder: Seeing within: molecular imaging of the cardiovascular system. Circ Res 94, 433-445 (2004)
45. Dorothy Sipkins, David Cheresh, Mahmood Kazemi, Linda Nevin, Mark Bednarski and King Li: Detection of tumor angiogenesis in vivo by alphavbeta3-targeted magnetic resonance imaging. Nat Med 4, 623-626 (1998)

46. Patrick Winter, Shelton Caruthers, Andrea Kassner, Thomas Harris, Lori Chinen, John Allen, Elizabeth Lacy, Huiying Zhang, J. David Robertson, Samuel Wickline and Gregory Lanza: Molecular imaging of angiogenesis in nascent $\mathrm{Vx}-2$ rabbit tumors using a novel alphavbeta3targeted nanoparticle and 1.5 tesla magnetic resonance imaging. Cancer Res 63, 5838-5843 (2003)

47. Anne Schmieder, Patrick Winter, Shelton Caruthers, Thomas Harris, Todd Williams, John Allen, Elizabeth Lacy, Huiying Zhang, Michael Scott, Grace Hu, J. David Robertson, Samuel Wickline and Gregory Lanza: Molecular MR imaging of melanoma angiogenesis with alphavbeta3-targeted paramagnetic nanoparticles. Magn Reson Med 53, 621-627 (2005)

48. Daniel J. Thorek, Antony Chen, Julie Czupryna and Andrew Tsourkas: Superparamagnetic iron oxide nanoparticle probes for molecular imaging. Ann Biomed Eng 34, 23-38 (2006)

49. Chunfu Zhang, Manfred Jugold, Eva Woenne, Twan Lammers, Bernd Morgenstern, Margareta Mueller, Hanswalter Zentgraf, Michael Bock, Michael Eisenhut, Wolfhard Semmler and Fabian Kiessling: Specific targeting of tumor angiogenesis by RGD-conjugated ultrasmall superparamagnetic iron oxide particles using a clinical 1.5T magnetic resonance scanner. Cancer Res 67, 1555-1562 (2007)

50. Xavier Montet, Karin Montet-Abou, Fred Reynolds, Ralph Weissleder and Lee Josephson: Nanoparticle imaging of integrins on tumor cells. Neoplasia 8, 214-222 (2006)

51. Iwona Wierzbicka-Patynowski, Stefan Niewiarowski, Cezary Marcinkiewicz, Juan Calvete, Mariola Marcinkiewicz and Mary Ann McLane: Structural Requirements of Echistatin for the Recognition of alpha vbeta 3 and alpha 5beta 1 Integrins. J Biol Chem 274, 37809-37814 (1999)

52. Dilantha Ellegala, Howard Leong-Poi, Joan Carpenter, Alexander Klibanov, Sanjiv Kaul, Mark Shaffrey, Jiri Sklenar and Jonathan Lindner: Imaging tumor angiogenesis with contrast ultrasound and microbubbles targeted to alpha (v)beta3. Circulation 108, 336-341 (2003)

53. Paul Dayton, David Pearson, Jarrod Clark, Scott Simon, Patricia Schumann, Reena Zutshi, Terry Matsunaga, Katherine Ferrara: Ultrasonic analysis of peptide- and antibody-targeted microbubble contrast agents for molecular imaging of alphavbeta3-expressing cells. Mol Imaging 3, 125-134 (2004)

54. Michaelann Shortencarier Tartis, Jennifer McCallan, Aaron Lum, Rachel LaBell, Susanne Stieger, Terry 
Matsunaga and Katherine Ferrara: Therapeutic effects of paclitaxel-containing ultrasound contrast agents. Ultrasound Med Biol 32, 1771-1780 (2006)

55. Vasilis Ntziachristos, A. G. Yodh, Mitchell Schnall and Britton Chance: Concurrent MRI and diffuse optical tomography of breast after indocyanine green enhancement. Proc Natl Acad Sci U S A 97, 2767-2772 (2000)

56. David Cuccia, Frederic Bevilacqua, Anthony Durkin, Sean Merritt, Bruce Tromberg, Gultekin Gulsen, Hon Yu, Jun Wang and Orhan Nalcioglu: In vivo quantification of optical contrast agent dynamics in rat tumors by use of diffuse optical spectroscopy with magnetic resonance imaging coregistration. Appl Opt 42, 2940-2950 (2003)

57. Christoph Bremer, Sebastian Bredow, Umar Mahmood, Ralph Weissleder and Ching-Hsuan Tung: Optical imaging of matrix metalloproteinase- 2 activity in tumors: feasibility study in a mouse model. Radiology 221, 523-529 (2001)

58. Xiaoyuan Chen, Peter Conti and Rex Moats: In vivo near-infrared fluorescence imaging of integrin alphavbeta3 in brain tumor xenografts. Cancer Res 64, 8009-8014 (2004)

59. Zhao-Hui Jin, Véronique Josserand, Stéphanie Foillard, Didier Boturyn, Pascal Dumy, Marie-Christine Favrot and Jean-Luc Coll: In vivo optical imaging of integrin alphaV-beta3 in mice using multivalent or monovalent cRGD targeting vectors. Mol Cancer 6, 41 (2007)

60. Angelika von Wallbrunn, Carsten Höltke, Michael Zühlsdorf, Walter Heindel, Michael Schäfers and Christoph Bremer: In vivo imaging of integrin alpha $\mathrm{v}$ beta 3 expression using fluorescence-mediated tomography. Eur J Nucl Med Mol Imaging 34, 745-754 (2007)

61. Weibo Cai, Andrew Hsu, Zi-Bo Li and Xiaoyuan Chen: Are quantum dots ready for in vivo imaging in human subjects? Nanoscale Res Lett 2, 265-281 (2007)

62. X. Michalet, F. F. Pinaud, L. A. Bentolila, J. M. Tsay, S. Doose, J. J. Li, G. Sundaresan, A. M. Wu, S. S. Gambhir and S. Weiss: Quantum dots for live cells, in vivo imaging, and diagnostics. Science 307, 538-544 (2005)

63. Igor Medintz, H. Tetsuo Uyeda, Ellen Goldman and Hedi Mattoussi: Quantum dot bioconjugates for imaging, labelling and sensing. Nat Mater 4, 435-446 (2005)

64. Weibo Cai, Dong-Woon Shin, Kai Chen, Olivier Gheysens, Qizhen Cao, Shan Wang, Sanjiv Gambhir and Xiaoyuan Chen: Peptide-labeled near-infrared quantum dots for imaging tumor vasculature in living subjects. Nano Lett 6, 669-676 (2006)
65. Richard Levenson: Spectral imaging and pathology: seeing more. Lab Med 35, 244-251 (2004)

66. James Mansfield, Kirk Gossage, Clifford Hoyt and Richard Levenson: Autofluorescence removal, multiplexing, and automated analysis methods for in-vivo fluorescence imaging. J Biomed Opt 10, 41207 (2005)

67. John Zimmer, Sang-Wook Kim, Shunsuke Ohnishi, Eichii Tanaka, John Frangioni, Moungi Bawendi: Size series of small indium arsenide-zinc selenide core-shell nanocrystals and application to in vivo imaging. $\mathrm{J} \mathrm{Am} \mathrm{Chem}$ Soc 128, 2526-2527 (2006)

68. Narayan Pradhan, David Battaglia, Yongcheng Liu and Xiaogang Peng: Efficient, stable, small, and watersoluble doped ZnSe nanocrystal emitters as non-cadmium biomedical labels. Nano Lett 7, 312-317 (2007)

69. Willem Mulder, Rolf Koole, Ricardo Brandwijk, Gert Storm, Patrick Chin, Gustav Strijkers, Celso de Mello Donegá, Klaas Nicolay and Arjan Griffioen: Quantum dots with a paramagnetic coating as a bimodal molecular imaging probe. Nano Lett 6, 1-6 (2006)

70. Roland Haubner, Wolfgang Weber, Ambros Beer, Eugenija Vabuliene, Daniel Reim, Mario Sarbia, KarlFriedrich Becker, Michael Goebel, Rüdiger Hein, HansJürgen Wester, Horst Kessler and Markus Schwaiger: Noninvasive visualization of the activated alphavbeta3 integrin in cancer patients by positron emission tomography and [18F]Galacto-RGD. PLoS Med 2, e70 (2005)

71. Ambros Beer, Roland Haubner, Michael Goebel, Stephan Luderschmidt, Mary Spilker, Hans-Jürgen Wester, Wolfgang Weber and Markus Schwaiger: Biodistribution and pharmacokinetics of the alphavbeta3 selective tracer $18 \mathrm{~F}$ Galacto-RGD in cancer patients. J Nucl Med 46, 13331341 (2005)

72. Ambros Beer, Roland Haubner, Ingo Wolf, Michael Goebel, Stephan Luderschmidt, Markus Niemeyer, AncaLigia Grosu, Maria-Jose Martinez, Hans Jürgen Wester, Wolfgang Weber and Markus Schwaiger: PET-based human dosimetry of $18 \mathrm{~F}$-galacto-RGD, a new radiotracer for imaging alphav beta3 expression. J Nucl Med 47, 763769 (2006)

73. I. Stangier, H.J. Wester, M. Schwaiger and A.J. Beer: Comparison of standardised uptake values and distribution volume for imaging of alphavbeta3 expression in breast cancer patients with [18F]Galacto-RGD PET. J Nucl Med 48 (S2) 406. (abstract) (2007)

74. Ambros Beer, Roland Haubner, Mario Sarbia, Michael Goebel, Stephan Luderschmidt, Anca Ligia Grosu, Oliver Schnell, Markus Niemeyer, Horst Kessler, Hans-Jürgen Wester, Wolfgang Weber and Markus Schwaiger: Positron emission tomography using [18F]Galacto-RGD identifies the level of integrin alphavbeta3 expresssion in man. Clin Cancer Res 12, 3942-3949 (2006) 
75. Ambros Beer, Anca-Ligia Grosu, Janette Carlsen, Andreas Kolk, Mario Sarbia, Isabelle Stangier, Petra Watzlowik, Hans-Jürgen Wester, Roland Haubner and Markus Schwaiger: [18F] Galacto-RGD PET for Imaging of alphavbeta3 Expression on Neovasculature in Patients with Squamous Cell Carcinoma of the Head and Neck. Clin Cancer Res 13, 6610-6616 (2007)

76. Ambros Beer, Sylvie Lorenzen, Stephan Metz, Ken Herrmann, Petra Watzlowik, Hans-Jürgen Wester, Christian Peschel, Florian Lordick and Markus Schwaiger: Comparison of integrin alphavbeta3 expression and glucose metabolism in primary and metastatic lesions in cancer patients: A PET study using [18F]Galacto-RGD and [18F]FDG. J Nucl Med 49, 22-29 (2008)

77. Tore Bach-Gansmo, Rimma Danielsson, Ariel Saracco, Brigitte Wilczek, Trond Bogsrud, Anne Fangberget, Åse Tangerud and Derek Tobin: Integrin receptor imaging of breast cancer: a proof-of-concept study to evaluate $99 \mathrm{mTc}$ NC100692. J Nucl Med 47, 1434-1439 (2006)

78. Ambros Beer, Markus Niemeyer, Janette Carlsen, Mario Sarbia, Jörg Nährig, Petra Watzlowik, Hans-Jürgen Wester, Nadia Harbeck and Markus Schwaiger: Patterns of alphavbeta3 Expression in Primary and Metastatic Human Breast Cancer as shown by [18F]Galacto-RGD PET. $J$ Nucl Med 49, 255-259 (2008)

Abbreviations: RGD: argine-glysine-aspartic acid tripeptide sequence; SPECT: single photon emission computed tomography; PET: positron emission tomography; MRI: magnetic resonance imaging; OI: optical imaging; US: ultrasound; ECM: extracellular matrix; HIF-1alpha: hypoxia inducible factor; VEGF: vascular endothelial growth vector; FMT: fluorescence mediated tomography; SSTR: somatostatin receptor; SAA: sugar amino acid; $\left[{ }^{18} \mathrm{~F}\right] \mathrm{NFP}$ 4-nitrophenyl-2$\left[{ }^{18}\right.$ F]fluoropropoinate; PEG: polyethylene glycol; n.c.a.: non-carrier added; SPIO: superparamagnetic iron oxide; USPIO: ultrasmall SPIO; IO: iron oxide; PASP: polyaspartic acid; PASP-IO: PASP-coated IO nanoparticles; RES: reticuloendothelial system; CEU: contrast enhanced ultrasound; AALs: acoustically active lipospheres; NIRF: near-infrared fluorescence; FMT: fluorescence mediated tomography; QD: quantum dots; $\mathrm{UV}$ : ultraviolet; $\mathrm{D}_{\mathrm{v}}$ : distribution volume; SUV: standard uptake value; SCCHN; squamous cell carcinoma of the head and neck; NSCLC: non small cell lung cancer.

Key Words: RGD Peptides, Molecular Imaging, alphavbeta3 Integrin, Angiogenesis, Noninvasive, Review

Send correspondence to: Hans-Jurgen Wester, Department of Nuclear Medicine, Klinikum rechts der Isar, Technische Universitat Munchen, Ismaninger Strasse 22, D - 81675 Munchen, Germany, Tel: 0049-89-41404586, Fax: 0049- 89-41404841, E-mail: H.J.Wester@1rz.tum.de

http://www.bioscience.org/current/vol14.htm 